\title{
83. ON THE PRODUCTION OF METEOR STREAMS BY COMETARY NUCLEI
}

\author{
L. A. KATASEV and N. V. KULIKOVA \\ Institute for Experimental Meteorology, Obninsk, U.S.S.R.
}

\begin{abstract}
An attempt is made to use Monte-Carlo techniques to model the process of formation of meteor streams. It is supposed that meteor streams are formed as the result of ejection of meteoroids from a cometary nucleus at perihelion. Possible ejection velocities are determined for the Draconids, Perseids, Leonids, and Taurids. In general the values do not exceed $100 \mathrm{~m} \mathrm{~s}^{-1}$. The data obtained can also be used to estimate the ages of the streams.
\end{abstract}

The ejection of dust by cometary nuclei is thought to be the main source of the meteoric particles forming the meteor streams. This ejection of meteoric material usually takes place in the vicinity of perihelion (Orlov, 1960; Vsekhsvyatskij, 1966; Southworth, 1963). This present work is an attempt, by the Monte-Carlo technique, to model the process of formation of meteor streams by the isotropic ejection of meteoric material from the nuclei of the parent comets when the latter are at perihelion.

Let us introduce a right-handed rectangular coordinate system $x y z$ having its origin at the cometary nucleus at perihelion, and with the $x$-axis along the prolonged radius vector of the comet, the $y$-axis in the direction of the comet's motion, and the $z$-axis perpendicular to the orbital plane. If $C$ is the velocity of ejection of a particle, and $C_{x}$, $C_{y}, C_{z}$ are its projections on the coordinate axes, we shall write

$$
\begin{aligned}
& C_{x}=C \sin \theta \cos \varphi \\
& C_{y}=C \sin \theta \sin \varphi \\
& C_{z}=C \cos \theta,
\end{aligned}
$$

where $\theta \in(0, \pi), \varphi \in(0,2 \pi)$. We define $C$ to be

$$
C=A \xi_{i},
$$

where $A$ is the upper limit of the ejection velocity, and $\xi_{i}$ is a random number in the range $(0,1)$.

The direction of the ejection of meteoroids depends on $\theta$ and $\varphi$, which can be found according to Neumann's (1951) formula:

$$
\begin{aligned}
\sin \varphi & =\frac{2 \xi_{i+1} \xi_{i+2} \zeta}{\xi_{i+1}^{2}+\xi_{i+2}^{2}} \\
\cos \varphi & =\frac{\xi_{i+1}^{2}-\xi_{i+2}^{2}}{\xi_{i+1}^{2}+\xi_{i+2}^{2}} \\
\cos \theta & =1-2 \xi_{i+3},
\end{aligned}
$$

where $\zeta=\left(\xi_{i+4}-\frac{1}{2}\right) /\left|\xi_{i+4}-\frac{1}{2}\right|= \pm 1$ defines the sign of $\sin \varphi$ with probability $1 / 2$ and $\xi_{i+1}, \xi_{i+2}, \xi_{i+3}, \xi_{i+4}$ are random numbers in $(0,1)$. 
In order to obtain the deviations of the orbital elements of the ejected particles from those of the parent-comet we shall use the formulae (Plavec, 1955):

$$
\begin{aligned}
\delta(1 / a) & =-2 v_{0} C_{y} \\
\delta P & =3 a_{0} P_{0} v_{0} C_{y} \\
\delta p & =2 q_{0}^{2} v_{0} C_{y} \\
\delta e & =2 q_{0} v_{0} C_{y} \\
\delta u & =C_{x} p_{0}^{1 / 2} e_{0}^{-1} \\
\tan i^{\prime} & =\left|C_{z}\right| v_{y}^{-1} \\
\delta i & =i^{\prime} \cos \omega_{0} \\
\delta \omega & =-\delta u-\delta \Omega \cos i_{0} \\
\delta \Omega & =i^{\prime} \sin \omega_{0} \operatorname{cosec} i_{0},
\end{aligned}
$$

where $a_{0}, P_{0}, q_{0}, e_{0}, i_{0}, \omega_{0}$ are the elements of the cometary orbit; $i, \Omega, \omega$ are the ecliptic angular elements of the meteor orbit; $u$ is the true anomaly, $p$ the parameter of the meteor orbit, $i^{\prime}$ the inclination of the meteor orbit to the orbital plane of the comet, $v_{0}$ the velocity of the comet at perihelion, and $v_{y}=v_{0}+C_{y}$ is the projection of the velocity of the ejected particle on the $y$-axis.

With the help of a high-speed computer we are able to obtain a great many possible orbits for meteoroids ejected with various velocities $C \leqslant A$ and at different angles. From the orbital elements of these particles it is possible to draw conclusions on the possibility of formation of specific meteor streams.

We have applied the procedure to the Perseid, Draconid, Leonid, and Taurid meteor streams (see Table I). For each variant no fewer than 200000 orbits were calculated. In the computation process the total interval of ejection velocity was divided into $K$ subintervals; in each subinterval the deviations of the orbital elements of the meteoroids were found according to Equation (4) and using Equations (1) to (3). The absolute values of these deviations were summed, and this sum was divided by $N$, where $N$ is the number of particles within the specified velocity interval.

For the formation of the Draconid meteor stream the velocity of ejection of meteoroids from the nucleus of P/Giacobini-Zinner should probably not exceed $50 \mathrm{~m} \mathrm{~s}^{-1}$. Deviations in the orbital elements of particles ejected at such velocities agree with the observational data for this stream in 1952 (Lovell, 1954). According to the observational data then the increments in eccentricity and semimajor axis were $\delta e=0.0013$, $\delta a=0.008 \mathrm{AU}$; we obtained such values at $C \leqslant 50 \mathrm{~m} \mathrm{~s}^{-1}$. The duration of the maximum of the 1952 shower was a little more than $30 \mathrm{~min}$, which corresponds to our result $\delta \Omega=1^{\prime} 20^{\prime \prime}$ at $C=50 \mathrm{~m} \mathrm{~s}^{-1}$.

The Leonid meteor shower, known from 889 (Astapovich, 1968), is still densely concentrated in an 18-AU arc of the orbit, the cross-section diameter there being $0.001 \mathrm{AU}$; about half the meteoroids are concentrated there (Murakami, 1961). From the observational data of the 1866 meteor shower Astapovich and Terent'eva 
TABLE I

Results of Monte-Carlo calculations on meteor streams

\begin{tabular}{llllr}
\hline$C\left(\mathrm{~m} \mathrm{~s}^{-1}\right)$ & $\delta P(\mathrm{yr})$ & $\delta a(\mathrm{AU})$ & $\delta e$ & $\delta \Omega$ \\
\hline Draconids & & & & \\
10 & 0.015 & 0.0050 & 0.00041 & 8.3 \\
30 & 0.043 & 0.016 & 0.0013 & 25.0 \\
50 & 0.074 & 0.026 & 0.0021 & 41.0 \\
100 & 0.15 & 0.052 & 0.0042 & 83.0 \\
200 & 0.30 & 0.10 & 0.0084 & 169.0 \\
& & & & \\
Leonids & & & & \\
0.025 & 0.00057 & 0.00012 & 0.0000011 & 0.034 \\
0.225 & 0.0053 & 0.0011 & 0.000010 & 0.31 \\
0.525 & 0.012 & 0.0026 & 0.000024 & 0.71 \\
1.025 & 0.24 & 0.0049 & 0.000045 & 1.42 \\
5.000 & 1.15 & 0.024 & 0.000219 & 6.67 \\
& & & & \\
Perseids & & & & \\
2.5 & 0.48 & 0.065 & 0.00011 & 0.35 \\
12.5 & 2.49 & 0.33 & 0.00055 & 1.75 \\
52.5 & 10.27 & 1.4 & 0.0023 & 7.37 \\
102.5 & 20.42 & 2.8 & 0.0045 & 14.03 \\
200.0 & 38.51 & 5.8 & 0.0086 & 27.21 \\
& & & & \\
Taurids & & & & \\
50 & 0.041 & 0.018 & 0.0012 & 35.0 \\
250 & 0.21 & 0.093 & 0.0064 & 180.0 \\
1000 & 0.87 & 0.43 & 0.027 & 742.0 \\
2000 & 1.68 & 1.1 & 0.052 & 1481.0 \\
3000 & 2.44 & 3.8 & 0.075 & 2104.0 \\
\hline & & & & \\
\hline
\end{tabular}

have calculated a new system of elements for the Leonids, which differ from the orbit of the parent comet $\mathrm{P} /$ Tempel-Tuttle in 1866 by $\delta a=0.00383 \mathrm{AU}, \delta e=0.000316$, $\delta P=0.07 \mathrm{yr}, \delta \Omega=3^{\prime}$. Variations in the character of particle motion obtained by modelling the ejection of meteoroids from the nucleus of $\mathrm{P} /$ Tempel-Tuttle show that the maximum velocity of ejection of material must probably not exceed $2.5 \mathrm{~m} \mathrm{~s}^{-1}$. The densest part of the stream could be formed by particles ejected with a velocity not greater than $25 \mathrm{~cm} \mathrm{~s}^{-1}$.

The differences between the Perseid orbit calculated by Southworth (1963) and the orbit of comet $1862 \mathrm{III}$ are $\delta a=2.68 \mathrm{AU}, \delta e=0.004, \delta P=19.5 \mathrm{yr}, \delta \Omega=48^{\prime \prime}$. We can obtain such deviations on the assumption of ejection velocities not greater than $100 \mathrm{~m} \mathrm{~s}^{-1}$. At $C=100 \mathrm{~m} \mathrm{~s}^{-1}$ the changes in the revolution period are such that the stream could extend all along the orbit after a few revolutions, coinciding with Southworth's conclusion that the age of the Perseids is $1000 \mathrm{yr}$. As for $\Omega$, it is small at all ejection velocities, and as Hamid (1950) pointed out, the stream is possibly influenced greatly by planetary perturbations.

It is supposed (Whipple and Hamid, 1952) that the Taurid meteor stream was formed as the result of ejection from the nucleus of $\mathrm{P} /$ Encke $4700 \mathrm{yr}$ ago and also 
$1500 \mathrm{yr}$ ago as subsequent ejection from a body having an orbit similar to that of the comet. The ejection velocity was $3 \mathrm{~km} \mathrm{~s}^{-1}$. The results obtained by modelling the formation process of the Taurids show that with an ejection velocity $C=3 \mathrm{~km} \mathrm{~s}^{-1}$ there would be substantial changes in semimajor axis $(\delta a=3.84 \mathrm{AU})$ and revolution period $(\delta P=2.45 \mathrm{yr})$. The investigations by Wright and Whipple (1950) give the following values for the orbital elements of the Northern and Southern Taurids, respectively:

$$
\begin{array}{llll}
a=2.14 \mathrm{AU}, & e=0.849, & P=3.13 \mathrm{yr}, \\
a=2.3 \mathrm{AU}, & e=0.7835, & P=3.49 \mathrm{yr} .
\end{array}
$$

For $\mathrm{P} /$ Encke these elements are $a=2.217 \mathrm{AU}, e=0.847, P=3.3 \mathrm{yr}$.

We obtain values for the orbital elements of ejected particles most appropriate to these data with velocities $C=250 \mathrm{~m} \mathrm{~s}^{-1}$, namely, $\delta a=0.09 \mathrm{AU}, \delta e=0.0004, \delta P=0.21$ yr. However, the heliocentric velocities acquired by the ejected particles at this ejection velocity do not correspond to the heliocentric velocities of the particles in the Northern and Southern Taurids. The necessary heliocentric velocities require ejection at velocities greater than $1 \mathrm{~km} \mathrm{~s}^{-1}$. Thus the question as to whether the Taurid meteors formed as the result of ejection from $\mathrm{P} /$ Encke is a complicated one, and to solve it we must consider the influence of planetary perturbations. Even at $C=250 \mathrm{~m} \mathrm{~s}^{-1}$ the changes in revolution period are such that in a few revolutions the stream could extend along the whole orbit.

In conclusion, we point out that in modelling the formation process of a meteor stream it is the revolution period and semimajor axis that are subjected to the greatest changes with increasing ejection velocity.

To evaluate the age of a stream Plavec (1955) has given the following formula:

$$
s P_{0}=\frac{\Delta M}{3 a_{0} v_{0} C_{y}},
$$

where $s P_{0}$ is the age of the stream in years, $\Delta M$ is the difference in mean anomaly, $a_{0}$ is the semimajor axis of the cometary orbit, $v_{0}$ is the heliocentric velocity of the comet at the point of ejection, and $C_{y}$ is the projection of the ejection velocity on the $y$-axis.

In the course of our calculations we automatically obtain the value of $C_{y}$ for every stream, but its uncertainty makes it difficult to use this formula directly. In this manner we found that the age of the Draconids is not greater than $60 \mathrm{yr}$. It is possible that this stream is still being formed at the present time. On the other hand, the age of the Leonids may be as much as $1580 \mathrm{yr}$.

\section{References}

Astapovich, I. S.: 1968, Probl. Kosmich. Fiz. No. 3, 103.

Hamid, S. E.: 1950, Ph.D. dissertation, Harvard University.

Lovell, B.: 1954, Meteor Astronomy, Clarendon Press, Oxford

Murakami, T.: 1961, Publ. Astron. Soc. Japan 13, 51, 212.

Neumann, J.: 1951, Natl. Bur. Sci. Appl. Math. Sci. 12, 36.

Orlov, S. V.: 1960, O Prirode Komet, Akad. Nauk SSSR.

Plavec, M.: 1955, Bull. Astron. Inst. Czech. 6, 20. 
Southworth, R. B.: 1963, Smithsonian Contr. Astrophys. 7, 299.

Vsekhsvyatskij, S. K.: 1966, Fizika Komet i Meteorov, Kiev, p. 32.

Whipple, F. L. and Hamid, S. E.: 1952, Bull. Roy. Obs. Helwan No. 41.

Wright, F. W. and Whipple, F. L.: 1950, Harvard Repr. Ser. 2 No. 35.

\section{Discussion}

F. L. Whipple: In my 1951 investigation I gave a formula for the distribution of ejection velocities, although without knowledge of the sizes of the particles it is difficult to use this formula. But it seems that the velocities amount to some tens of metres per second.

B. Yu. Levin: The velocities obtained from Whipple's formula and by other methods are less than those obtained in this investigation. This is probably because perturbations have not been taken into account here.

$V$. V. Fedynskij: What value did you adopt for the upper limit of the ejection velocities?

$N$. V. Kulikova: It was different for each stream and determined according to the best representation of the observations.

L. M. Shul'man: The creation of a meteor stream is not an instantaneous act, but it takes place over a definite period of time. Further, the ejection cannot be considered isotropic.

N. V. Kulikova: I agree. We intend in future to make a more sophisticated formulation of the problem that takes these points into account. 